\title{
ORIGINAL RESEARCH \\ Value of Coronal Reformations in the CT Evaluation of Acute Head Trauma
}

S.C. Wei

S. Ulmer

M.H. Lev

S.R. Pomerantz

R.G. González

J.W. Henson

BACKGROUND AND PURPOSE: Routine axial CT images may not be ideally suited for detecting ICH in transversely oriented locations such as the floor of the anterior and middle cranial fossas and vertex. This study was performed to evaluate whether coronal reformations improve detection of ICH in NCCT performed for head trauma.

MATERIALS AND METHODS: All patients undergoing a first NCCT in the ED for evaluation of head trauma were included prospectively during a 6-month interval. NCCT images were reconstructed into standard 5-mm axial datasets and were also reformatted into coronal datasets of 5-mm sections and 2.5-mm intervals. Thirty-two of $213(15 \%)$ scans were interpreted as showing traumatic ICH. These cases were interspersed with 30 studies without $\mathrm{ICH}$. Cases were reviewed for the presence and location of $\mathrm{ICH}$ by 2 staff neuroradiologists.

RESULTS: Of 213 patients, 32 NCCTs demonstrated ICH (a total of 104 foci). Fifteen of 104 (14\%) ICHs (8 patients) were detected solely on coronal images. Locations included the floor of the anterior and middle cranial fossas, vertex, corpus callosum, falx, tentorium, and occipital convexity. Coronal reformations allowed exclusion of suspicious findings on axial images in 14 instances (7 patients) Coronal images aided interpretation in 29/104 (28\%) findings.

CONCLUSIONS: Coronal reformations improve the detection of $\mathrm{ICH}$ over axial images alone, especially for lesions that lie in the axial plane immediately adjacent to bony surfaces. The use of coronal reformations should be considered in the routine interpretation of head $\mathrm{CT}$ examinations performed for the evaluation of head trauma.

ABBREVIATIONS: $\mathrm{ED}=$ emergency department; $\mathrm{ICH}=$ intracranial hemorrhage; $\mathrm{NCCT}=$ noncontrast cranial CT

A pproximately 1.4 million people sustain a traumatic brain injury each year in the United States. ${ }^{1}$ Because of its ready availability, speed, and cost-effectiveness, $\mathrm{CT}$ of the head has become the imaging technique of choice for diagnosing neurocranial traumatic lesions. ${ }^{2}$ Since the invention of CT in 1971 by Sir Godfrey Hounsfield, CT imaging has vastly improved from time-intensive single-section scanning to the present multidetector row $\mathrm{CT}$, allowing acquisition of isotropic datasets with a voxel dimension of $\leq 0.6 \mathrm{~mm}$. The availability of this volumetric data has heralded the development of exciting new data-processing techniques, including maximum intensity projections, volume-rendered $3 \mathrm{D}$ reformations, and multiplanar reformations.

These new techniques have been rapidly incorporated into daily image interpretation in many aspects of neuroradiology, such as neurovascular and spine imaging. However, the workhorse imaging study of neuroradiology, the NCCT of the head, remains surprisingly unchanged and may underuse these modern imaging capabilities.

Multiplanar reformats have been shown to aid in interpretation of other CT studies such as abdominopelvic examina-

Received April 22, 2009; accepted after revision July 3

From the Division of Neuroradiology (S.C.W., S.U., M.H.L. S.R.P., R.G.G.) and Division of Neuroradiology and Neurology Service (J.W.H.), Massachusetts General Hospital, Boston, Massachusetts.

Stephen C. Wei and Stephan Ulmer contributed equally to this study.

Please address correspondence to John W. Henson, MD, and Michael H. Lev, MD, Division of Neuroradiology, Massachusetts General Hospital, 55 Fruit St, Boston, MA 02114; e-mail: john.henson@swedish.org and mlev@partners.org

DOI 10.3174/ajnr.A1824

tions, in which they have been demonstrated to provide additional information in $17 \%-23 \%$ of studies. ${ }^{3}$ Since the mid1990s, multiplanar reformations have been found to be invaluable in the CT examination of trauma patients for the evaluation of the facial bones ${ }^{4}$ and cervical spine $e^{5}$ and are now considered standard components of these examinations. They are also routinely used in other neuroradiologic CT examinations, such as temporal bone CT studies. ${ }^{6}$

To our knowledge, no study to date has investigated the use of multiplanar reformations with regard to NCCT examinations. Therefore, this study was performed to determine the utility of coronal CT reformations in the CT evaluation of head trauma. In particular, the study attempted to determine whether coronal reformations aid in the detection and characterization of ICH.

\section{Materials and Methods}

\section{Patients}

The study population comprised all patients in the ED during a 6-month period referred for initial NCCT to evaluate head trauma of any cause. Patients with a prior CT for trauma (eg, those with an initial study from an outside institution) were excluded. The protocol was approved by the institutional review board.

\section{Scanning Procedure}

CT images were acquired on a 16-section LightSpeed scanner (GE Healthcare, Milwaukee, Wisconsin) by using a $140-\mathrm{kV}$ and $220-\mathrm{mAs}$ technique. Images were reconstructed into a contiguous 5-mm axial dataset by using a standard algorithm. A second near-isotropic $1.25-\mathrm{mm}$ axial dataset was also reconstructed and subsequently refor- 
matted into a coronal image set of 5-mm thick sections at a $2.5-\mathrm{mm}$ reconstruction interval, also by using a standard algorithm.

\section{Image Analysis}

As part of routine clinical care, neuroradiology fellows or staff neuroradiologists interpreted both axial and coronal images at the time the NCCT was initially performed. All cases that demonstrated ICH were selected during a continuous 6-month period. These were added to an equivalent number of NCCT examinations without ICH, inserted in random order as controls to create a final enriched study set of examinations for subsequent review.

The enriched study set was analyzed in a secondary review by 2 staff neuroradiologists in consensus, and the presence and location of each separate focus of hemorrhage were recorded. Axial images were reviewed first. Six weeks later, this review was followed by a separate one of the coronal images only, with readers blinded to the results of the initial axial series review. The complete axial and coronal studies were then reviewed together for a final assessment regarding the presence and location of ICH.

\section{Results}

NCCT studies of 213 patients were analyzed. This group included 134 male patients (63\%) and 79 female patients (37\%), with a mean age of 46 years (range, 3-92 years). The indication for NCCT was a "fall" in 107 patients (50\%; 61 male, 46 female), "motor vehicle crash" in 68 (32\%; 45 male, 23 female), "assault" in 18 ( $8 \% ; 17$ male, 1 female), "direct head trauma" in 16 ( $8 \%$; 8 male, 8 female), and "unknown" in 4 (2\%; 3 male, 1 female).

Among the 213 patients, 32 (14.3\%) had ICH documented in the initial ED interpretation. On blinded secondary review, axial or coronal images suggested the presence of ICH in 104 separate locations in these 32 patients ( 81 potential foci detected on axial images; 89 potential foci, on coronal images). Side-by-side comparison of axial and coronal images reduced this number of ICH to 80 true-positive foci. The difference of 24 findings on axial or coronal series alone (14 axial, 10 coronal) consisted of those findings initially interpreted as ICH but later judged to be negative for ICH on combined review of both image series.

Therefore, 14/104 (13\%) findings on axial image review alone were determined to represent false-positives when the combined image sets were reviewed. For 5/14 of these falsepositives, the consensus final interpretation was confirmed as negative by follow-up CT. In the remaining 9/14 cases, no confirmation was possible (no follow-up CT was performed).

ICH was detected solely on coronal images for 15/104 (14\%) hemorrhagic foci, which was present on $8 / 32$ NCCT examinations (25\%). These included 7 subdural hematomas, 5 subarachnoid hemorrhages, and 3 parenchymal hemorrhages (contusions and diffuse axonal injury). The locations of the 15 additional ICHs detected on coronal reformations included the floor of anterior cranial fossa $(n=6)$, middle cranial fossa $(n=3)$, vertex $(n=2)$, corpus callosum $(n=1)$, falx $(n=1)$, tentorium $(n=1)$, and occipital convexity $(n=1)$. For $11 / 15$ of these axial false-negatives, the consensus final interpretation was confirmed as positive (by follow-up CT in 10 foci and at surgery in 1 focus). In the remaining 4/15 patients, no confirmation was possible (no coronal reformation was performed, 4 foci).
The subset of findings, including false-positives excluded and true-positives detected, represents a total of 29/104 findings $(28 \%)$ in which coronal images aided interpretation.

Twenty-three $(23 / 32,72 \%)$ patients demonstrated multiple foci of hemorrhage. The remaining 9 patients $(9 / 32,28 \%)$ represented those in whom only a single focus of hemorrhage was identified on either axial or coronal images. Because the presence or absence of a single finding may be more likely to impact patient care and/or disposition, these cases were further reviewed.

Of these 9 patients in whom only a single focus of $\mathrm{ICH}$ was found, 2 were determined on final review (coronal and axial) to represent true-positives $(2 / 9,22 \%)$. One of these $(1 / 2,50 \%)$ was detected on both the independent coronal and axial series reviews, and $1(1 / 2,50 \%)$ was detected on an independent coronal series review alone. The 2 positive findings were confirmed at surgery ( 1 patient) and on follow-up CT (1 patient). The remaining $7 / 9$ cases $(78 \%)$ were determined on final review to represent false-positives, $3 / 7$ (43\%) suggested on axial series and 4/7 (57\%) on coronal series.

Of the 32 patients demonstrating ICH, 2 (2/32, 6\%) underwent craniotomy for ICH or elevated intracranial pressure. In these 2 patients, use of the coronal image set was not considered crucial in the decision to operate.

\section{Discussion}

Even though MR imaging may be more sensitive for detection of small lesions after traumatic head injury, ${ }^{7} \mathrm{CT}$ remains the technique of choice for the emergent evaluation of brain trauma due to its wide availability, ability to assess less stable patients, and capability to rapidly detect and characterize intracranial lesions that may require immediate neurosurgery. ${ }^{8}$ Although coronal reformations require only minutes to create, ${ }^{9}$ in our institution typically $<1$ minute by using currently available software and a streamlined postprocessing protocol, they are not routinely part of most noncontrast cranial CT imaging protocols.

Our data demonstrate that coronal reformations are valuable in the detection and characterization of traumatic ICH. There were 104 sites of ICH identified on either axial or coronal images in this study, of which 80 were determined to be true-positive lesions on final review of both coronal and axial series. Of these 104 possible sites of $\mathrm{ICH}$, the most instructive were the 15 true-positive lesions not detected on axial images (15/104 [14\%] in 8 patients) and the 14 false-positive findings first suggested on axial images but later excluded on coronal images (14/104 in 10 patients).

These 29 findings total more than one-fourth of all findings and represent those that were solely detected or excluded by using coronal reformations. After we examined these cases, it became clear that axial images may be less accurate in the detection of intracranial hemorrhage in areas that are parallel to the axial image plane, especially those immediately adjacent to bony surfaces, where volume averaging artifacts may further obscure small foci of ICH. The limitations of axial images for evaluating these areas are of concern, because these are precisely the locations that may be at greater risk for injury in some mechanisms of head trauma. ${ }^{10}$

In our study, the 2 most common locations for a false- 

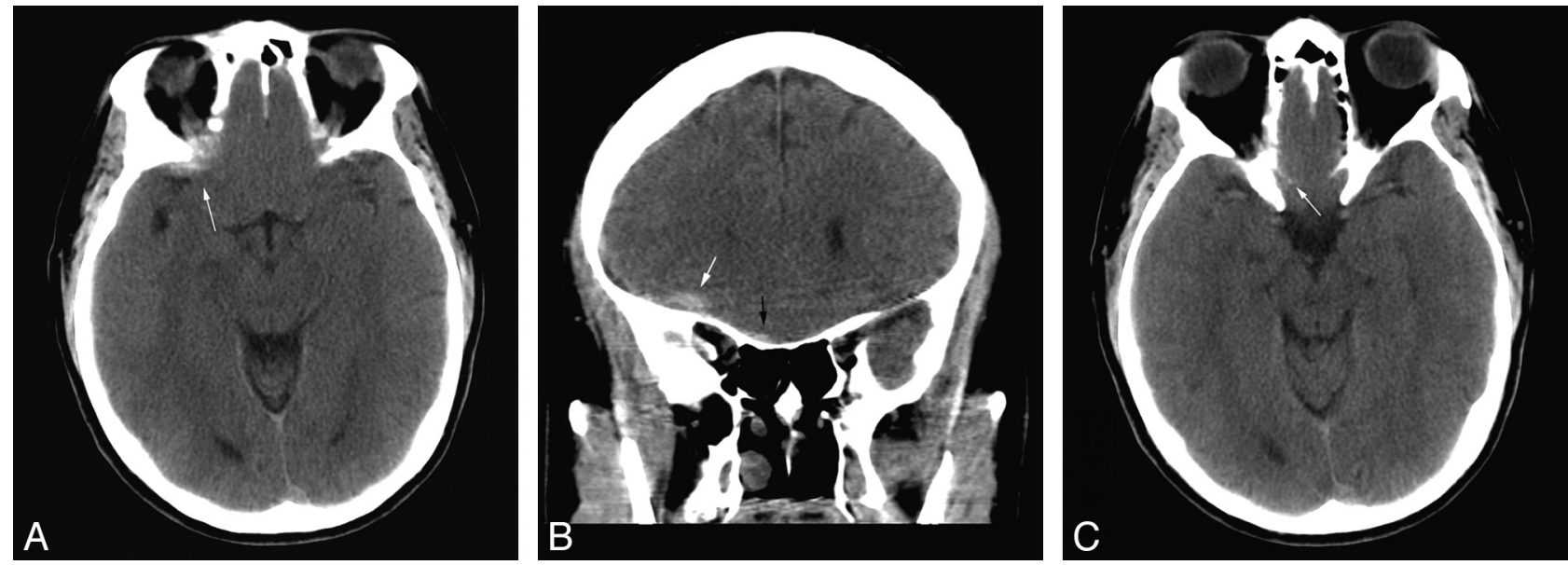

Fig 1. A, A small hemorrhagic contusion (white arrow) is partially obscured by volume averaging with the adjacent bone. $B$, The same lesion is more conspicuous and confidently identified on coronal reformations (white arrow). A small right subdural hematoma (black arrow) is noted. $C$, On the corresponding axial image, no abnormality can be identified in the region of the small right subdural hematoma (small white arrow).
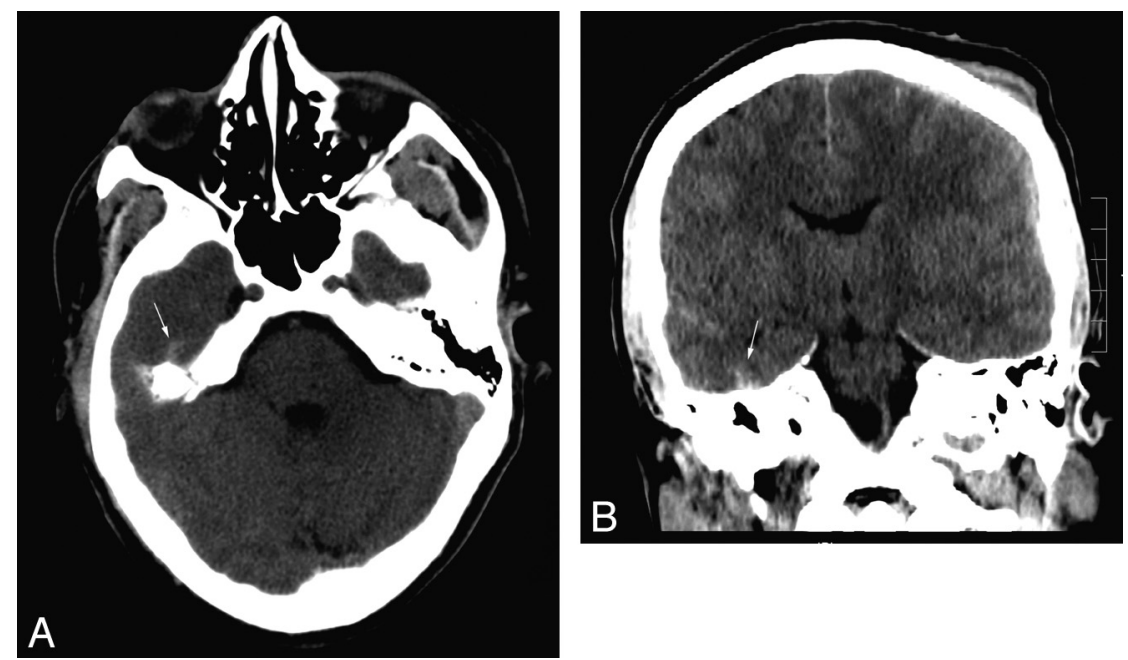

Fig 2. A, Hyperattenuation (arrow) in the superficial right temporal lobe is partially obscured by volume averaging through the temporal bone. $B$, A clear focus of hemorrhagic contusion (arrow) is seen on the corresponding coronal image.

negative finding on axial images were the floor of the anterior cranial fossa $(n=6)$ and the floor of the middle cranial fossa $(n=3)$, a total of $9 / 15(60 \%)$ of the false-negatives from the independent axial review. Figures 1 and 2 demonstrate small hemorrhagic contusions along the floor of the right anterior and middle cranial fossas. On the axial series, these are partially obscured by volume averaging from the immediately adjacent bones. The ICH is subtly visible on the axial image (Fig 1A) but is much more conspicuous on the corresponding coronal image (Fig $1 B$ ). A small right subdural hematoma is detectable only on the coronal image set (Fig $1 B$ ) and cannot be identified on the corresponding axial image (Fig 1C). A similar image pair shown in Fig 2 demonstrates a right inferior temporal contusion, partially obscured by volume averaging through the adjacent temporal bone on the axial image set but clearly seen on coronal images.

The addition of coronal images as a second orthogonal imaging set also helps to detect findings oriented along these same orthogonal planes. For example, detection of short thin transversely oriented lesions can be difficult on axial images due to volume averaging through the adjacent normal pa- renchyma, which tends to obscure the typically low-contrast findings. Figure 3 demonstrates a focus of parenchymal hemorrhage in the corpus callosum, which is much more conspicuous on coronal images.

Additionally, lesions that are vertically oriented may also be more easily detected on coronal reformations than on axial images. Because the long dimension of the finding will be oriented along the coronal axis, a greater proportion of the lesion is seen on each single coronal section, which can increase conspicuity of the lesion. Figure 4 shows a small vertically oriented anterior falcine subdural hematoma. On the axial image set, this is a relatively subtle finding. However, it is easily detected on coronal reformations.

One additional advantage of the coronal images is that the second imaging plane increases specificity by enabling more confident evaluation of questionable findings detected on axial images. Figure 5 shows a potential lesion initially detected on individual axial image review. Subsequent review of the corresponding coronal images shows correlative normal findings, permitting the confident exclusion of pathology.

We found that coronal images also added value when one is 

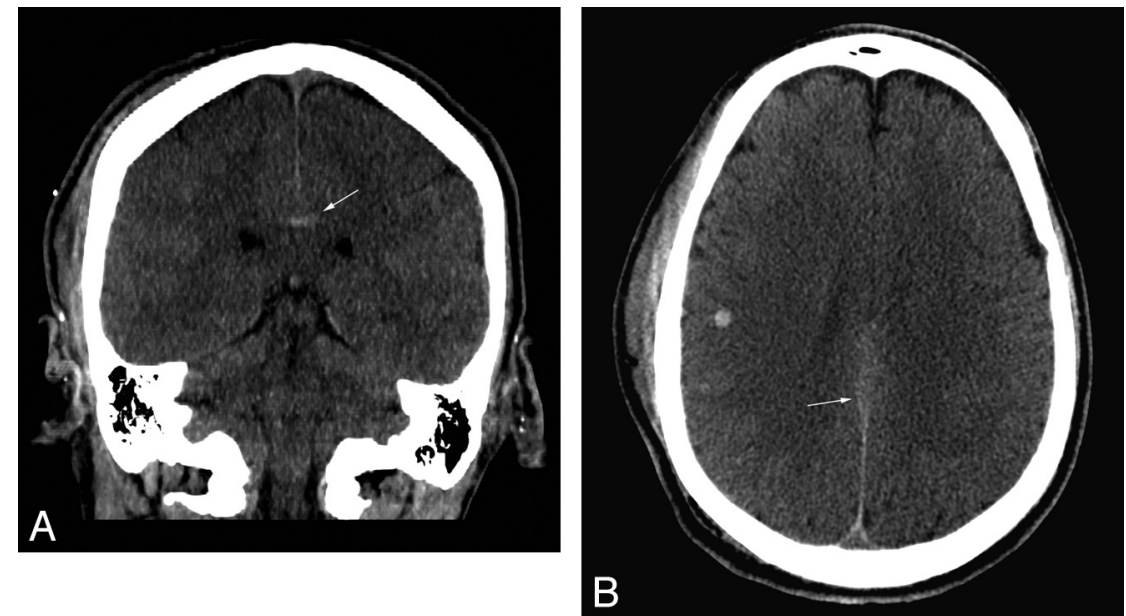

Fig 3. $A$, A small focus of parenchymal hemorrhage (arrow) is clearly seen in the corpus callosum on the coronal reformation. $B$, The lesion (arrow) is much more subtly apparent on the corresponding axial image.
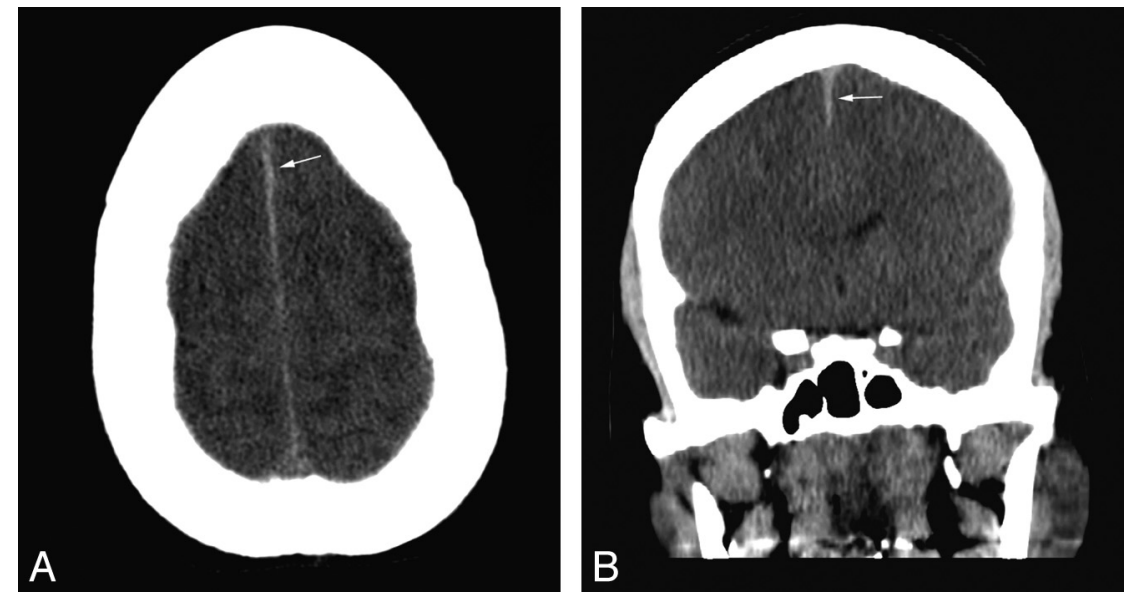

Fig 4. A, Minimal thickening of the anterior falx (arrow) is not prospectively identified on initial review of the axial images. $B$, This is confirmed to represent a small subdural hematoma (arrow) on the corresponding coronal image.
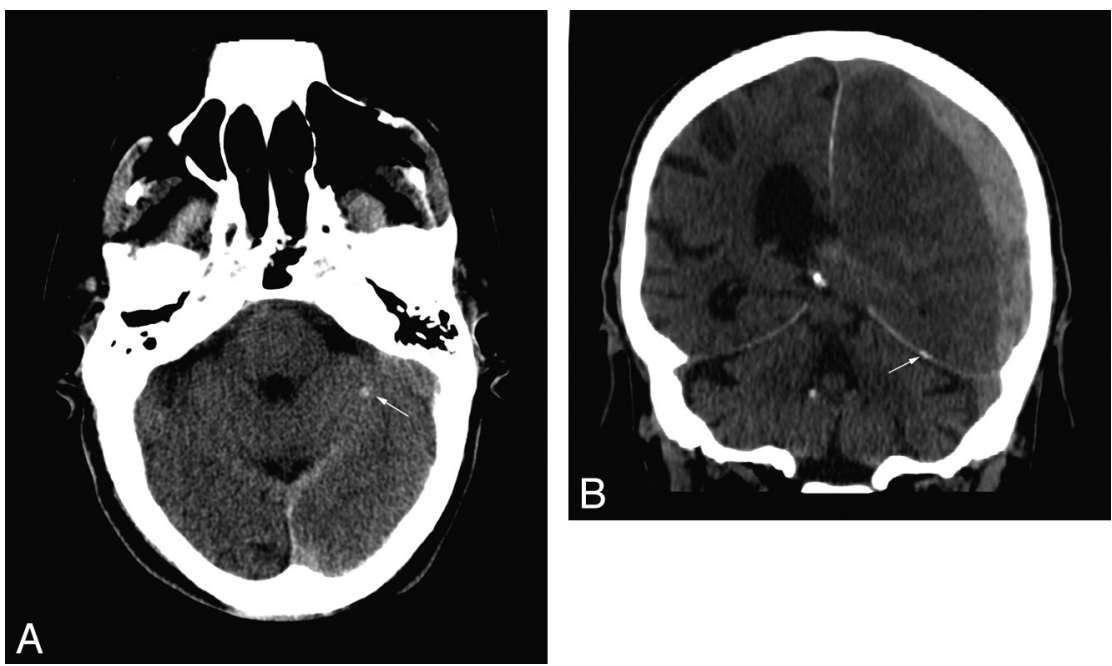

Fig 5. A, Hyperattenuated focus (arrow) in the left cerebellum interpreted as suspicious for parenchymal hemorrhage. $B$, Coronal images show that the finding represents tentorial calcification (arrow).

imaging features of head trauma other than ICH. Some findings may be nearly or completely invisible in the axial plane. Figure 6 shows a tiny focus of pneumocephalus adjacent to the left temporal bone, which is nearly completely obscured by the bordering mastoid air cells on the axial set but is clearly seen to be intracranial on the coronal image. Similarly, Fig 7 demon- 

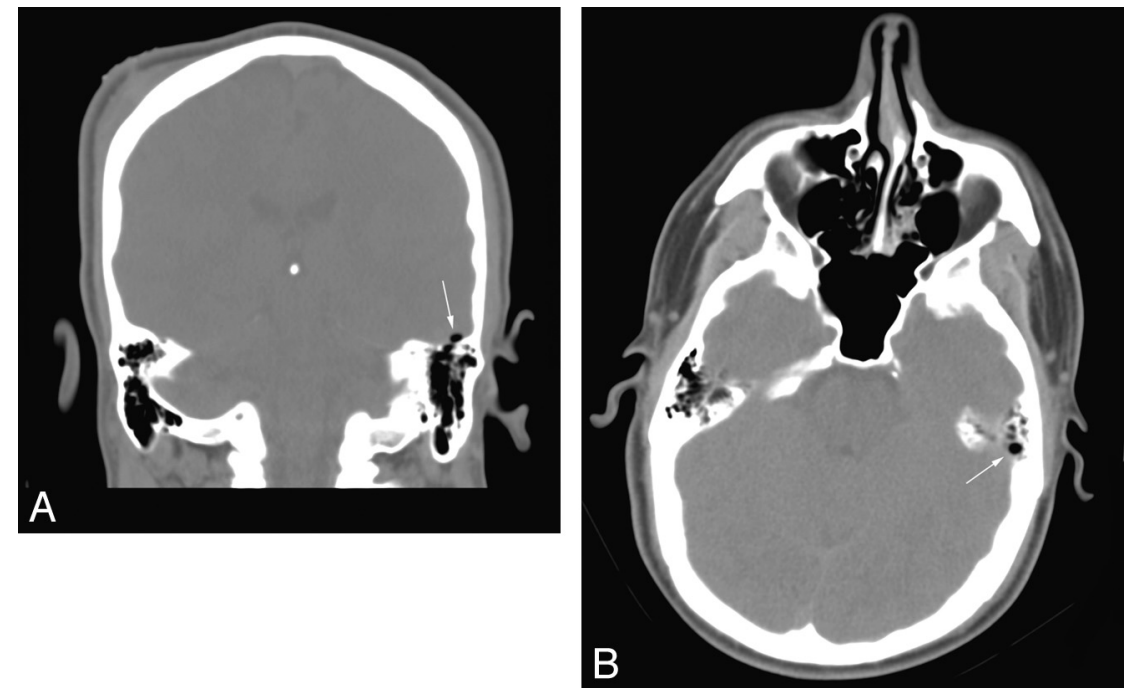

Fig 6. $A$, A small focus of pneumocephalus (arrow) is related to a nondisplaced fracture through the left temporal bone. $B$, On the axial images, the findings are obscured due to volume averaging through neighboring mastoid air cells (arrow).

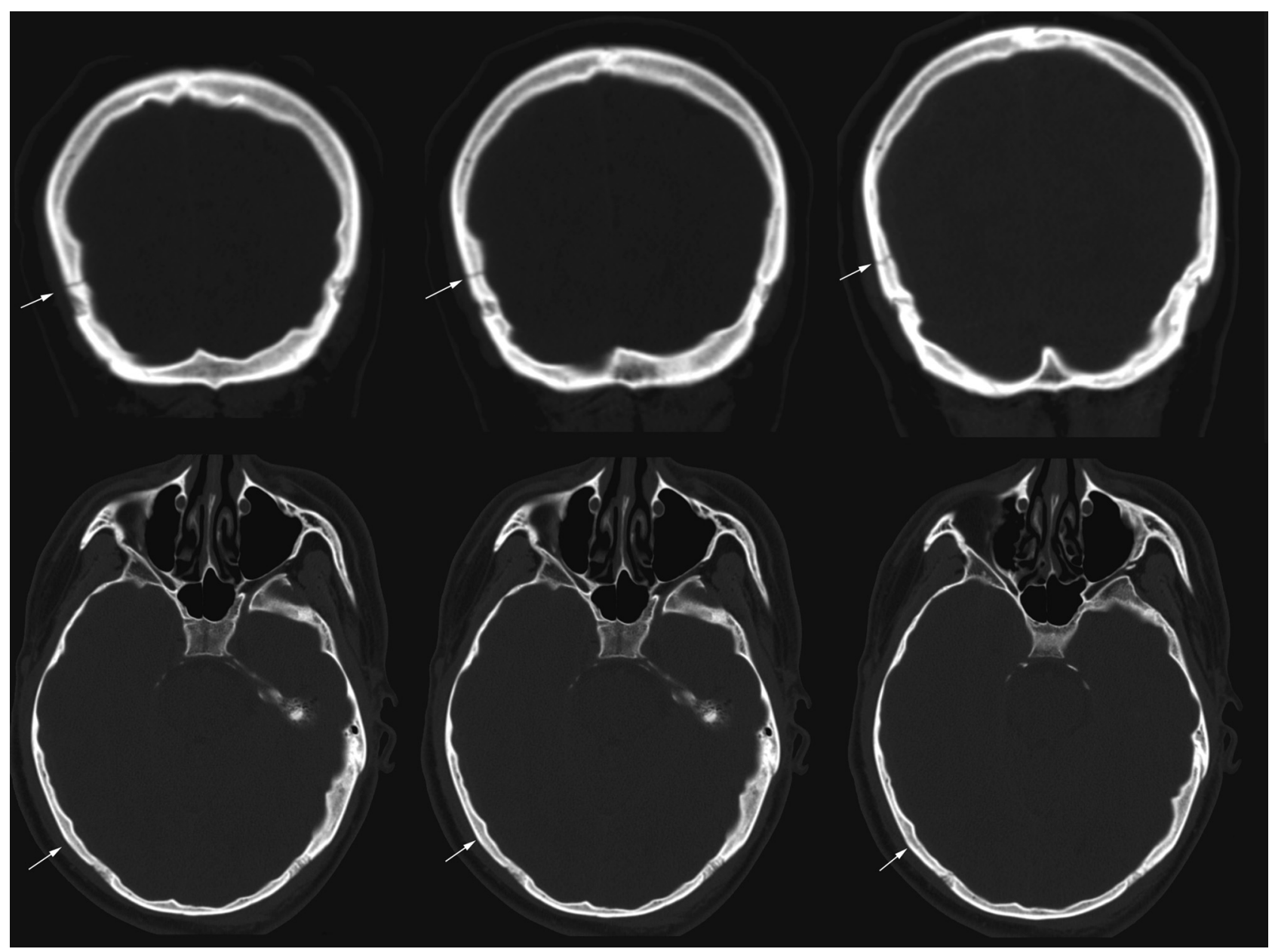

Fig 7. Three coronal images show a tranverse nondisplaced fracture (arrows) through the right parietal bone. The corresponding axial images show no evidence of fracture (arrows).

strates that horizontal skull fractures, when they occur parallel to the acquisition plane, may be wholly invisible on axial images.

The value of coronal images was particularly clear for the 9 patients $(9 / 213,4 \%$ of the study population) whose NCCT showed only 1 finding, for which accurate evaluation of that single finding is even more relevant. Although these changes in interpretation did not alter the requirement for surgery, such findings can significantly influence medical care. For example, patients in whom subtle ICH is detected should be appropriately monitored and are likely to be re-imaged after an ap- 
propriate interval, rather than discharged with a follow-up outpatient appointment. ${ }^{11}$ Similarly, detection of subtle pneumocephalus indicates the presence of open head trauma, for which antibiotic therapy is often given as a meningitis prophylaxis. $^{12}$

One potential limitation of the study is the size of 213 patients ( 32 with positive findings). However, the distribution of the trauma mechanism in this study is reasonably similar to that in larger published studies - for example, Smits et al, ${ }^{2}$ suggesting that it is a satisfactory representative section of head trauma ED presentations.

\section{Conclusions}

In the evaluation of noncontrast cranial CT, coronal reformations improved detection and characterization for the evaluation of ICH compared with standard axial images alone. Of 213 patients in this study, coronal images detected an additional $14 \%$ of hemorrhagic foci involving 8 patients (4\%) and allowed the exclusion of ICH initially suggested on axial images in 7 patients (3\%).

The advantages of evaluating coronal images become especially clear for lesions that lie in the axial plane immediately adjacent to bony surfaces (the anterior cranial fossa, middle cranial fossa, vertex). Coincidentally, these may also be locations that are at higher risk for injury in head trauma. In this study, several foci of ICH oriented transversely in the axial plane were found to be completely invisible on axial images alone.

Because coronal reformations can be simply and rapidly constructed from typical NCCT data acquisitions and provide significant benefit for interpretation of these studies, they have become a component of the routine protocol for NCCT at our institution. On the basis of the results of this study, we suggest consideration of their use in the routine interpretation of NCCT examinations, especially in those performed for the evaluation of head trauma.

\section{References}

1. Langlois JA, Rutland-Brown W, Thomas KE. Traumatic Brain Injury in the United States: Emergency Department Visits, Hospitalizations, and Deaths. Atlanta: Centers for Disease Control and Prevention, National Center for Injury Prevention and Control; 2006. Available at: http://www.cdc.gov/ncipc/tbi/ TBI.htm. Accessed April 2009

2. Smits M, Dippel DW, de Haan GG, et al. External validation of the Canadian CT Head Rule and the New Orleans Criteria for CT scanning in patients with minor head injury. JAMA 2005;294:1519-25

3. Sandrasegaran K, Rydberg J, Tann M, et al. Benefits of routine use of coronal and sagittal reformations in multi-slice CT examination of the abdomen and pelvis. Clin Radiol 200:62:340-47

4. Fox LA, Vannier MW, West OC, et al. Diagnostic performance of CT, MPR and 3DCT imaging in maxillofacial trauma. Comput Med Imaging Graph 1995; 19:385-95

5. Rubenstein D, Escotte EJ, Mestek MF. Computed tomographic scans of minimally displaced type II odontoid fractures. J Trauma 1996;40:204-10

6. Mazziotti S, Arceri F, Vinci S, et al. Role of coronal oblique reconstruction as a complement to CT study of the temporal bone: normal anatomy. Radiol Med 2006;111:607-17

7. Mittl RL, Grossman RI, Hiehle JF, et al. Prevalence of MR evidence of diffuse axonal injury in patients with mild head injury and normal head CT findings. AJNR Am J Neuroradiol 1994;15:1583-89

8. Eisenberg HM, Gary HE Jr, Aldrich EF, et al. Initial CT findings in 753 patients with severe head injury: a report from the NIH Traumatic Coma Data Bank. J Neurosurg 1990;73:688-98

9. Heyer CM, Rduch GJ, Wick M, et al. Evaluation of multiple trauma victims with 16-row multidetector CT (MDCT): a time analysis [in German]. Rofo 2005; 177:1677-82

10. Hardman JM, Manoukian A. Pathology of head trauma. Neuroimaging Clin NAm 2002;12:175-87

11. Sifri ZC, Homnick AT, Vaynman A, et al. A prospective evaluation of the value of repeat cranial computed tomography in patients with minimal head injury and an intracranial bleed. $J$ Trauma 2006;61:862-67

12. Villalobos T, Arango C, Kubilis P, et al. Antibiotic prophylaxis after basilar skull fractures: a meta-analysis. Clin Infect Dis 1998;27:364-69 